\title{
Principles for Cartogram Design, Elicited from Manual Construction of Cartograms for the 50 U.S. States
}

\author{
Barry J Kronenfeld \\ Eastern Illinois University - barrykronenfeld@ gmail.com
}

Keywords: Cartograms, Cartographic Design, Shapes, Perception

\begin{abstract}
:
Value-by-area cartograms provide a unique and intuitive way to visualize count/quantity variables such as human population. While much research into cartograms has focused on their algorithmic construction, manually constructed cartograms are often favored for their aesthetic quality and recognizability. There is a lack of research, however, into the principles guiding cartogram design. Such principles would assist artistically minded cartographers and algorithm developers alike.

A series of population cartograms was designed by the author to support visualization of the Covid-19 pandemic via the Covid-19 Open Visualization (COViz) project (https://www.eiu.edu/gisci/coviz). These included a cartogram of the USA by state and of each U.S. state and the territory of Puerto Rico by county or equivalent administrative unit. All cartograms were produced using Cartogram Studio, a free software tool created by the author that supports manual design of continuous cartograms. Cartogram design was performed over the course of 18 months, with each individual cartogram requiring approx. $10 \mathrm{hrs}$ on average to complete to satisfaction. A sample of five original maps and corresponding cartograms designed for the project are shown in Figure 1. Cartograms produced by the diffusion algorithm using the free ScapeToad software are also shown for comparison.

The process of manual cartogram design afforded the opportunity to formulate general principles to guide the process. Developing these principles served a couple of purposes. First, their specification provided procedural memory, reducing the creative burden of what would otherwise have been a series of unrelated artistic projects. This also engendered a certain degree of standardization of style across the different cartograms in the series. Second, the principles were intended to support design of more visually appealing and functionally recognizable cartograms. Since ease of district identification and aesthetic appeal are well-known factors influencing the effectiveness of cartograms, these principles may serve as a reference to the cartographic community.

The list of cartogram design principles presented here is incomplete and should not be construed as the definitive last word on the matter, but rather as an initial proposal to be debated and discussed. The principles capture aspects of cognitive and perceptual preference as well as strategies for handling the sometimes extreme demands of the cartogram definitional constraint. Many parallels also exist between what follows and general cartographic design principles, gestalt theory, etc. Some ideas derive from prior cartograms produced by others and feedback from friends and colleagues.
\end{abstract}

Principle 1: Simplify, Simplify, Simplify

A cartogram is a thematic map with a very narrow purpose. It may not even be perceived as a map per se, but rather as a "mapical" graphic representation of data. Since users need only to recognize districts and not features within districts, and since shape deformation leads to a very distracting visual panoply, real-world features should be simplified as much as possible. Boundary features (e.g. bends, protrusions) should never be added, and all but the bare minimum required for recognizability should be removed. If complete removal is deemed unjustifiable, bends and jags may be softened to the point where they may be used for recognition purposes but do not distract from the overall structure. Greater simplification is warranted for sparsely populated districts since they will shrink on the cartogram, reducing their discernability.

Examples: In the USA cartogram (Fig. 1a) the upper peninsula of Michigan is greatly simplified due to its size reduction. Several bends have been removed from the coastlines of North and South Carolina, Florida and Louisiana.

\section{Principle 2: If Necessary, Schematize}

Where population constraints demand extreme deformation that precludes shape recognition, it may be useful to schematize districts. Schematization may involve geometric regularization, exaggeration of broad features and grouping and alignment of multiple features. 

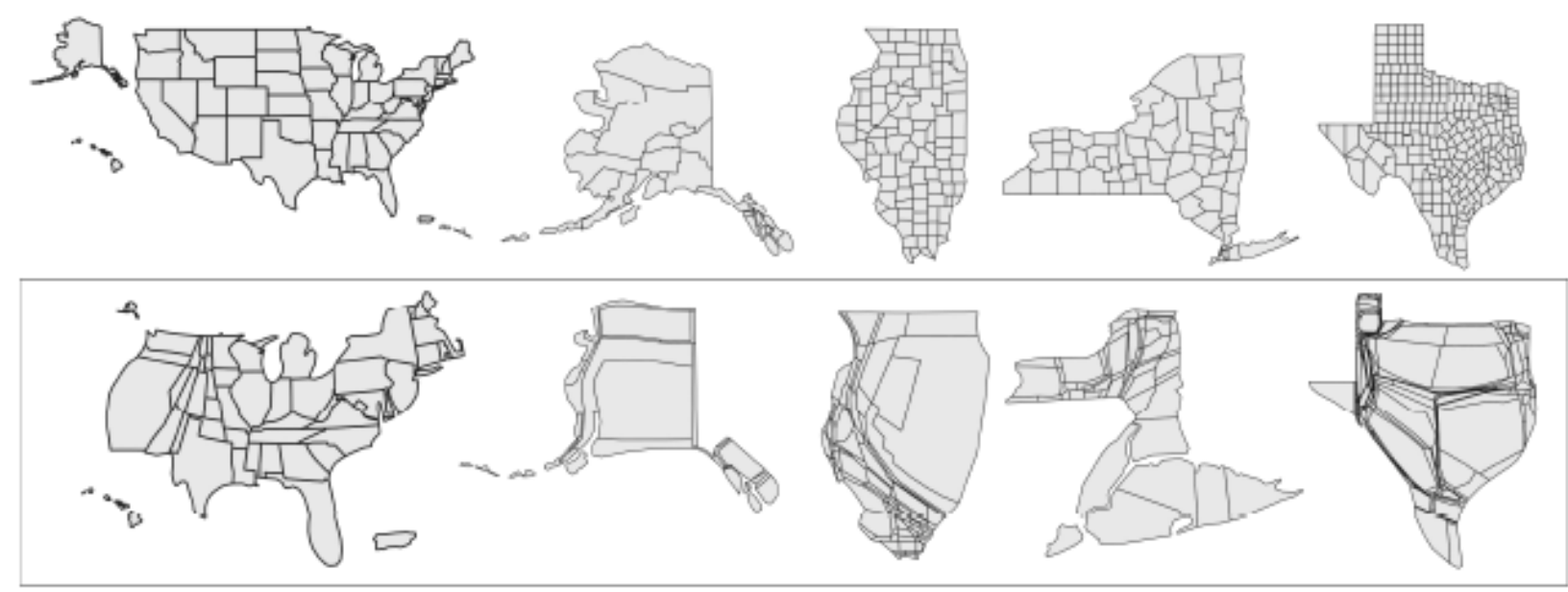

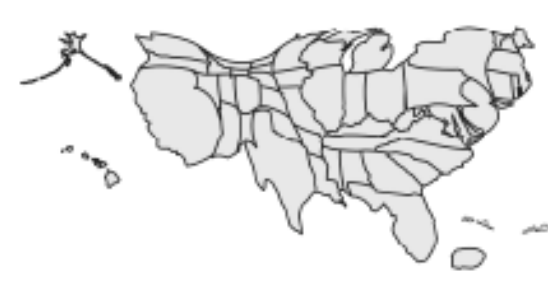

(a)

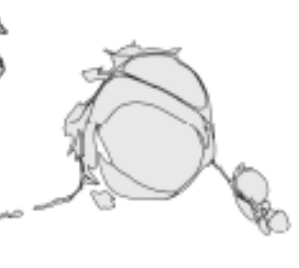

(b)

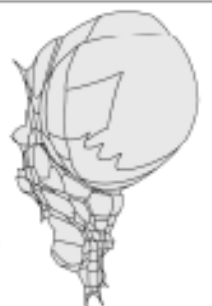

(c)

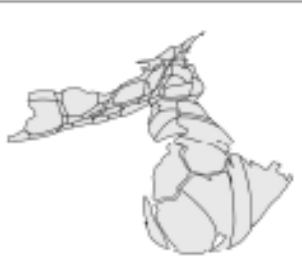

(d)

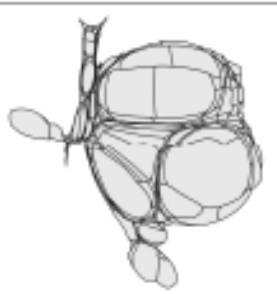

(e)

Figure 1. Original maps (top), cartograms designed manually by the author (middle), and cartograms produced by the diffusion algorithm (bottom). Areas of districts are proportional to 2018 population. (a) USA, (b) Alaska, (c) Illinois, (d) New York, (e) Texas.

Examples: The coastline of California (Fig. 1a) is reduced to a broad curve that is more recognizable than the individual bays and capes. In Alaska (Fig. 1b), the most populated counties are transformed into loosely rectangular shapes. These are aligned with each other, as are the surrounding smaller-population counties

\section{Principle 3: Preserve Shapes Hierarchically}

Not all shapes are equal. In most cases the shape of the map's outer boundary (i.e. the overall region) should have highest priority, followed by the most densely populated and geographically distinct districts. These will form landmarks (anchors) that can be used to locate and identify other districts. Note that priority is not absolute, but requires a balance. It is not usually advisable to maintain the shape of the outer boundary exactly as this will introduce extreme distortion in some places.

Examples: The overall shape of Illinois (Fig. 1c) is more important than that of its individual counties, which are unfamiliar to most people anyway. A population cartogram of New York (Fig. 1d) requires extreme enlargment of the downstate region in comparison to upstate that precludes preservation of the state's overall distinctive shape, but the general shapes of these two regions can be separately preserved.

\section{Principle 4: Maintain Boundary Continuation}

If a series of boundaries forms a linear continuation then this should be preserved as much as possible. Continuation can be maintained by minimizing angularity at boundary points and aligning sequential boundary sections into a line or broad arc. This principle borrows from the concept of stroke continuation in network generalization. It may be applied loosely, including series of borders that are not strictly linear or not completely connected.

Examples: On the USA cartogram (Fig. 1a), the boundaries between the following pairs of states form a broad continuation from east to west: Virginia/North Carolina, Kentucky/Tennessee, Missouri/Iowa, Kansas/Oklahoma, Colorado/New Mexico, Utah/Arizona. The same is true for the southern border of the northern tier of Illinois counties (Fig. 1c).

\section{Principle 5: Isolate Disconnected Parts}

As a practical matter, islands can be easily moved and resized without any shape deformation at all. The same is true to a lesser degree for peninsulas. Bodies of water can be used as a sink for unwanted distortion.

Examples: The shapes of Puerto Rico (Fig. 1a), Manhattan and Staten Island (Fig. 1d) can be preserved exactly, and the same is essentially true for peninsular Florida (Fig. 1a). By shifting Michigan's disconnected upper peninsula to the west (Fig. 1b), shape distortion is reduced throughout the upper midwest. 